\title{
Recent Results on a Generalization of the Laplacian ${ }^{\dagger}$
}

\author{
A.B. SIMAS $^{1 *}$ and F.J. VALENTIM ${ }^{2}$ \\ Received on May 14, 2014 / Accepted on May 23, 2015
}

\begin{abstract}
In this paper we discuss recent results regarding a generalization of the Laplacian. To be more precise, fix a function $W\left(x_{1}, \ldots, x_{d}\right)=\sum_{k=1}^{d} W_{k}\left(x_{k}\right)$, where each $W_{k}: \mathbb{R} \rightarrow \mathbb{R}$ is a right continuous with left limits and strictly increasing function. Using $W$, we construct the generalized laplacian $\mathcal{L}_{W}=$ $\sum_{i=1}^{d} \partial_{x_{i}} \partial_{W_{i}}$, where $\partial_{W_{i}}$ is a generalized differential operator induced by the function $W_{i}$. We present results on spectral properties of $\mathcal{L}_{W}$, Sobolev spaces induced by $\mathcal{L}_{W}$ (W-Sobolev spaces), generalized partial differential equations, generalized stochastic differential equations and stochastic homogenization.
\end{abstract}

Keywords: $W$-Sobolev space, generalized Laplacian, homogenization, partial differential equations.

\section{INTRODUCTION}

In the '50s William Feller introduced a more general concept of differential operators, that is, operators of the type $(d / d W)(d / d V)$ where, typically, $W$ and $V$ are strictly increasing functions with $V$ (but not necessarily $W$ ) being continuous. In this paper we are interested in the formal adjoint of $(d / d W)(d / d V)$, which is simply $(d / d V)(d / d W)$, in the case $V(x)=x$ is the identity function. For more details on Feller's operators, we refer the reader to [4, 5, 9].

Recently, some attention has been raised to a class of generalized differential operator involving the derivative with respect to a strictly increasing function $W$, we cite $[2,7,6,8,9,11,12]$ as some examples of this fact. On one hand, this operator can be naturally obtained from behavior of some interacting particle systems with random conductances with the interesting feature that, in contrast with usual homogenization phenomena, the entire noise survives in the limit and the differential operator itself depends on the specific realization of random conductance [2, 7]. On the other hand, a second surprising aspect is that the differential equation that appears in the limit is a second-order differential operator in which one of the derivatives is a derivative with respect to function $W$, which may have jumps. Even more, the set of jumps of $W$ can be dense in $\mathbb{R}$.

\footnotetext{
*Corresponding author: Alexandre B. Simas

$\dagger$ Research supported by CNPq and FAPES.

${ }^{1}$ Departamento de Matemática, Universidade Federal da Paraíba, Cidade Universitária, Campus I, 58051-970 João Pessoa, PB, Brasil. E-mail: alexandre@mat.ufpb.br

2 Departamento de Matemática, Universidade Federal do Espírito Santo, Av. Fernando Ferrari, 514, Goiabeiras, 29075-910, Vitória, ES, Brasil. E-mail: fabio.valentim@ufes.br
} 
The goal of this paper is present an overview of the main recent results regarding this differential operator. The rest of the paper unfolds as follows: in Section 2 we present the generalized Laplacian, which we denote by $\mathcal{L}_{W}$; in Section 3 we construct the $W$-Sobolev spaces and present several properties that are analogous to classical results on Sobolev spaces, we also present results on existence and uniqueness for $W$-generalized elliptic equations, and a uniqueness result for $W$-generalized parabolic equations; in Section 4 we present stochastic homogenization results of suitable random operators $\nabla^{N} A^{N} \nabla_{W}^{N}$, that are discretizations of the operator $\mathcal{L}_{W}$.

This paper is essencially inspired in works $[11,12,3,13]$.

\section{THE OPERATOR $\mathcal{L}_{W}$}

Fix a function $W: \mathbb{R}^{d} \rightarrow \mathbb{R}$ such that

$$
W\left(x_{1}, \ldots, x_{d}\right)=\sum_{k=1}^{d} W_{k}\left(x_{k}\right)
$$

where $W_{k}: \mathbb{R} \rightarrow \mathbb{R}$ are strictly increasing right continuous functions with left limits (càdlàg), and periodic in the sense that

$$
W_{k}(u+1)-W_{k}(u)=W_{k}(1)-W_{k}(0)
$$

for all $u \in \mathbb{R}$ and $k=1, \ldots, d$. To keep notation simple, we assume that $W_{k}$ vanishes at the origin, that is, $W_{k}(0)=0$.

Denote by $\mathbb{T}$ the unidimensional torus and $\langle\cdot, \cdot\rangle$ the inner product of $L^{2}(\mathbb{T})$ :

$$
\langle f, g\rangle=\int_{\mathbb{T}} f(u) g(u) d u .
$$

For each $k=1,2, \ldots, d$ let $\mathcal{D}_{W_{k}}$ be the set of functions $f$ in $L^{2}(\mathbb{T})$ such that

$$
f(x)=a+b W_{k}(x)+\int_{(0, x]} W_{k}(d y) \int_{0}^{y} \mathfrak{f}(z) d z
$$

for some function $\mathfrak{\uparrow}$ in $L^{2}(\mathbb{T})$ such that

$$
\int_{0}^{1} \mathfrak{f}(z) d z=0, \quad \int_{(0,1]} W_{k}(d y)\left\{b+\int_{0}^{y} \mathfrak{f}(z) d z\right\}=0
$$

Define the operator $\mathcal{L}_{W_{k}}: \mathcal{D}_{W_{k}} \rightarrow L^{2}(\mathbb{T})$ as $\mathcal{L}_{W_{k}} f=\mathfrak{f}$. Formally,

$$
\mathcal{L}_{W_{k}} f=\frac{d}{d x} \frac{d}{d W_{k}} f
$$

where the generalized derivative $d / d W_{k}$ is defined as

$$
\frac{d f}{d W_{k}}(x)=\lim _{\epsilon \rightarrow 0} \frac{f(x+\epsilon)-f(x)}{W_{k}(x+\epsilon)-W_{k}(x)},
$$

if the above limit exists and is finite. 
By a convenient restriction of the operators $\mathcal{L}_{W_{k}}$ to a dense subspace $\mathcal{D}_{k} \subset \mathcal{D}_{W_{k}}$, it is not difficult to prove that $\mathcal{L}_{W_{k}}: \mathcal{D}_{k} \rightarrow L^{2}(\mathbb{T})$ is symmetric and non-positive. Thus, by using Friedrichs extension (see, for instance, [14, chapter 5]), one obtains that the extended operator, also denoted by $\mathcal{L}_{W_{k}}, \mathcal{L}_{W_{k}}: \mathcal{D}_{W_{k}} \rightarrow L^{2}(\mathbb{T})$, is self-adjoint and, the set $\mathcal{A}_{W_{k}}$ of the eigenvectors of $\mathcal{L}_{W_{k}}$ forms a complete orthonormal system in $L^{2}(\mathbb{T})$, the details of this approach can be found in [6].

Let

$$
\mathcal{A}_{W}=\left\{f: \mathbb{T}^{d} \rightarrow \mathbb{R} ; f\left(x_{1}, \ldots, x_{d}\right)=\prod_{k=1}^{d} f_{k}\left(x_{k}\right), f_{k} \in \mathcal{A}_{W_{k}}\right\},
$$

and define the operator $\mathbb{L}_{W}: \mathbb{D}_{W}:=\operatorname{span}\left(\mathcal{A}_{W}\right) \rightarrow L^{2}\left(\mathbb{T}^{d}\right)$ as follows: for $f=\prod_{k=1}^{d} f_{k} \in$ $\mathcal{A}_{W}$,

$$
\mathbb{L}_{W}(f)\left(x_{1}, \ldots x_{d}\right)=\sum_{k=1}^{d} \prod_{j=1, j \neq k}^{d} f_{j}\left(x_{j}\right) \mathcal{L}_{W_{k}} f_{k}\left(x_{k}\right),
$$

and extend to $\mathbb{D}_{W}$ by linearity. In particular, $\mathbb{D}_{W}$ is dense in $L^{2}\left(\mathbb{T}^{d}\right)$; and the set $\mathcal{A}_{W}$ forms a complete, orthonormal, countable system of eigenvectors for the operator $\mathbb{L}_{W}$.

Let $L_{x^{k} \otimes W_{k}}^{2}\left(\mathbb{T}^{d}\right)$ be the Hilbert space of measurable functions $H: \mathbb{T}^{d} \rightarrow \mathbb{R}$ such that

$$
\int_{\mathbb{T}^{d}} d\left(x^{k} \otimes W_{k}\right) H(x)^{2}<\infty,
$$

where $d\left(x^{k} \otimes W_{k}\right)=d x_{1} \cdots d x_{k-1} d W_{k} d x_{k+1} \cdots d x_{d}$.

Lemma 2.1. Let $f, g \in \mathbb{D}_{W}$, then for $i=1, \ldots, d$,

$$
\int_{\mathbb{T}^{d}}\left(\partial_{x_{i}} \partial_{W_{i}} f(x)\right) g(x) d x=-\int_{\mathbb{T}^{d}}\left(\partial_{W_{i}} f\right)\left(\partial_{W_{i}} g\right) d\left(x^{i} \otimes W_{i}\right) .
$$

In particular,

$$
\int_{\mathbb{T}^{d}} \mathbb{L}_{W} f(x) g(x) d x=-\sum_{i=1}^{d} \int_{\mathbb{T}^{d}}\left(\partial_{W_{i}} f\right)\left(\partial_{W_{i}} g\right) d\left(x^{i} \otimes W_{i}\right) .
$$

that is, $\mathbb{L}_{W}$ is symmetric and non-positive.

The proof consist in an application of Fubini's theorem and an approximation of the integral by Riemann sum. Informally,

$$
\begin{aligned}
\int_{\mathbb{T}}\left(\partial_{x_{i}} \partial_{W_{i}} f\right) g d x_{i} & =-\int_{\mathbb{T}}\left(\partial_{W_{i}} f\right)\left(\partial_{x_{i}} g\right) d x_{i} \approx-\sum \frac{\Delta f}{\Delta W_{i}} \frac{\Delta g}{\Delta x_{i}} \Delta x_{i} \\
& =-\sum \frac{\Delta f}{\Delta W_{i}} \frac{\Delta g}{\Delta W_{i}} \Delta W_{i} \approx-\int_{\mathbb{T}}\left(\partial_{W_{i}} f\right)\left(\partial_{W_{i}} g\right) d W_{i}
\end{aligned}
$$

where the sum is over partitions of the torus $\mathbb{T}$ and $\Delta h$ stands for the increment of the function $h$ with respect to the partition. The details can be found in [13]. 
Consider

$$
\mathcal{D}_{W}=\left\{v=\sum_{k=1}^{\infty} v_{k} h_{k} \in L^{2}\left(\mathbb{T}^{d}\right) ; \sum_{k=1}^{\infty} v_{k}^{2} \alpha_{k}^{2}<+\infty\right\},
$$

where $h_{k} \in \mathcal{A}_{W}$ and $\alpha_{k}$ is the eigenvalue associated to to eigenvector $h_{k}$.

Define the operator $\mathcal{L}_{W}: \mathcal{D}_{W} \rightarrow L^{2}\left(\mathbb{T}^{d}\right)$ by

$$
-\mathcal{L}_{W} v=\sum_{k=1}^{+\infty} \alpha_{k} v_{k} h_{k}
$$

The operator $\mathcal{L}_{W}$ is clearly an extension of the operator $\mathbb{L}_{W}$, and formally,

$$
\mathcal{L}_{W} f=\sum_{k=1}^{d} \mathcal{L}_{W_{k}} f
$$

where

$$
\mathcal{L}_{W_{k}} f=\partial_{x_{k}} \partial_{W_{k}} f
$$

and the partial generalized derivative $\partial_{W_{k}}$ is defined by

$$
\partial_{W_{k}} f(x)=\lim _{\epsilon \rightarrow 0} \frac{f\left(x_{1}, \ldots, x_{k}+\epsilon, \ldots, x_{d}\right)-f\left(x_{1}, \ldots, x_{k}, \ldots, x_{d}\right)}{W_{k}\left(x_{k}+\epsilon\right)-W_{k}\left(x_{k}\right)},
$$

if the above limit exists and is finite. The following theorem gives us some properties of the operator $\mathcal{L}_{W}$.

Theorem 2.2. The operator $\mathcal{L}_{W}: \mathcal{D}_{W} \rightarrow L^{2}\left(\mathbb{T}^{d}\right)$ enjoys the following properties:

i) The domain $\mathcal{D}_{W}$ is dense in $L^{2}\left(\mathbb{T}^{d}\right)$. In particular, the set of eigenvectors $\mathcal{A}_{W}$ forms a complete orthonormal system;

ii) The eigenvalues of the operator $-\mathcal{L}_{W}$ form a countable set $\left\{\alpha_{k}\right\}_{k \geq 0}$. All eigenvalues have finite multiplicity, and it is possible to obtain a re-enumeration $\left\{\alpha_{k}\right\}_{k \geq 0}$ such that

$$
0=\alpha_{0} \leq \alpha_{1} \leq \cdots \text { and } \lim _{n \rightarrow \infty} \alpha_{n}=\infty
$$

iii) The operator $\mathbb{I}-\mathcal{L}_{W}: \mathcal{D}_{W} \rightarrow L^{2}\left(\mathbb{T}^{d}\right)$ is bijective;

iv) $\mathcal{L}_{W}: \mathcal{D}_{W} \rightarrow L^{2}\left(\mathbb{T}^{d}\right)$ is self-adjoint and non-positive:

$$
\left\langle-\mathcal{L}_{W} f, f\right\rangle \geq 0
$$

v) $\mathcal{L}_{W}$ is dissipative.

In view of i), iii) and iv) we may use Hille-Yosida theorem to conclude that $\mathcal{L}_{W}$ is the generator of a strongly continuous contraction semigroup $\left\{P_{t}: L^{2}\left(\mathbb{T}^{d}\right) \rightarrow L^{2}\left(\mathbb{T}^{d}\right)\right\}_{t \geq 0}$. 
We will now provide an outline of the proof. The details can be found in [13]. Since $\mathbb{D}_{W} \subset \mathcal{D}_{W}$, we have that $\mathcal{D}_{W}$ is dense in $L^{2}\left(\mathbb{T}^{d}\right)$. The properties of the eingevalues follows from properties of $\mathcal{L}_{W_{k}}$ and the definition of $\mathcal{L}_{W}$. From ii) we have that $\mathbb{I}-\mathcal{L}_{W}$ is injective. For $v \in L^{2}\left(\mathbb{T}^{d}\right)$, we have that

$$
v=\sum_{k=1}^{+\infty} v_{k} h_{k} \in L^{2}\left(\mathbb{T}^{d}\right), \quad \text { where the } v_{k} \text { are such that } \sum_{k=1}^{\infty} v_{k}^{2}<+\infty
$$

Then,

$$
u=\sum_{k=1}^{+\infty} \frac{v_{k}}{\gamma_{k}} h_{k} \in \mathcal{D}_{W},
$$

and $\left(\mathbb{I}-\mathcal{L}_{W}\right) u=v$. Hence $\mathbb{I}-\mathcal{L}_{W}$ is bijective.

Let $\mathcal{L}_{W}^{*}: \mathcal{D}_{W^{*}} \subset L^{2}\left(\mathbb{T}^{d}\right) \rightarrow L^{2}\left(\mathbb{T}^{d}\right)$ be the adjoint of $\mathcal{L}_{W}$. Since $\mathcal{L}_{W}$ is symmetric, we have $\mathcal{D}_{W} \subset \mathcal{D}_{W^{*}}$. So, to show that $\mathcal{L}_{W}=\mathcal{L}_{W}^{*}$, it is enough to show that $\mathcal{D}_{W^{*}} \subset \mathcal{D}_{W}$. Let $\varphi=$ $\sum_{k=1}^{+\infty} \varphi_{k} h_{k} \in \mathcal{D}_{W^{*}}$ be given, and let $\mathcal{L}_{W *} \varphi=\psi \in L^{2}\left(\mathbb{T}^{d}\right)$. Then, for all $v=\sum_{k=1}^{+\infty} v_{k} h_{k} \in$ $\mathcal{D}_{W}$,

$$
\langle v, \psi\rangle=\left\langle v, \mathcal{L}_{W *} \varphi\right\rangle=\left\langle\mathcal{L}_{W} v, \varphi\right\rangle=\sum_{k=1}^{+\infty}-\alpha_{k} v_{k} \varphi_{k} .
$$

Hence $\psi=\sum_{k=1}^{+\infty}-\alpha_{k} \varphi_{k} h_{k}$. In particular, $\sum_{k=1}^{+\infty} \alpha_{k}^{2} \varphi_{k}^{2}<+\infty$ and $\varphi \in \mathcal{D}_{W}$. Thus, $\mathcal{L}_{W}$ is self-adjoint. By ii) $\mathcal{L}_{W}$ is non-positive.

Finally, fix a function $g$ in $\mathcal{D}_{W}$, let $\lambda>0$, and let also $f=\left(\lambda \mathbb{I}-\mathcal{L}_{W}\right) g$. So

$$
\lambda\langle g, g\rangle+\left\langle-\mathcal{L}_{W} g, g\right\rangle=\langle g, f\rangle \leq\langle g, g\rangle^{1 / 2}\langle f, f\rangle^{1 / 2} .
$$

Using iv), the second term on the left hand side is non-negative. Thus, $\|\lambda g\| \leq\|f\|=\|(\lambda \mathbb{I}-$ $\left.\mathcal{L}_{W}\right) g \|$, that is, $\mathcal{L}_{W}$ is dissipative.

\section{W-SOBOLEV SPACE AND DIFFERENTIAL EQUATIONS}

We construct the $W$-Sobolev spaces, which consist of functions $f$ having weak generalized gradients

$$
\nabla_{W} f=\left(\partial_{W_{1}} f, \ldots, \partial_{W_{d}} f\right) .
$$

Several properties, that are analogous to classical results on Sobolev spaces, are obtained. Existence and uniqueness results for $\mathrm{W}$-generalized elliptic equations, and uniqueness results for $\mathrm{W}$-generalized parabolic equations are also established. More details on these results can be found in [11]

\subsection{The definition and properties}

Recall the definition of Hilbert space $L_{x^{k} \otimes W_{k}}^{2}\left(\mathbb{T}^{d}\right)$ given in Section 2. 
Let $L_{x^{i} \otimes W_{i}, 0}^{2}\left(\mathbb{T}^{d}\right)$ be the closed subspace of $L_{x^{i} \otimes W_{i}}^{2}\left(\mathbb{T}^{d}\right)$ consisting of the functions that have zero mean with respect to the measure $d\left(x^{j} \otimes W_{j}\right)$ :

$$
\int_{\mathbb{T}^{d}} f d\left(x^{i} \otimes W_{i}\right)=0 .
$$

We define the Sobolev space of $W$-generalized derivatives as the space of functions $g \in L^{2}\left(\mathbb{T}^{d}\right)$ such that for each $i=1, \ldots, d$ there exists a fuction $G_{i} \in L_{x^{i} \otimes W_{i}, 0}^{2}\left(\mathbb{T}^{d}\right)$ satisfying the following integral by parts identity for every function $f \in \mathbb{D}_{W}$ :

$$
\int_{\mathbb{T}^{d}}\left(\partial_{x_{i}} \partial_{W_{i}} f\right) g d x=-\int_{\mathbb{T}^{d}}\left(\partial_{W_{i}} f\right) G_{i} d\left(x^{i} \otimes W_{i}\right) .
$$

We denote this space by $H_{1, W}\left(\mathbb{T}^{d}\right)$. For each function $g \in H_{1, W}$ we denote $G_{i}$ by $\partial_{W_{i}} g$, and we call it the $i$ th generalized weak derivative of the function $g$ with respect to $W$.

In [11] it is shown that the set $H_{1, W}\left(\mathbb{T}^{d}\right)$ is a Hilbert space with respect to the inner product

$$
\langle f, g\rangle_{1, W}=\langle f, g\rangle+\sum_{i=1}^{d} \int_{\mathbb{T}^{d}}\left(\partial_{W_{i}} f\right)\left(\partial_{W_{i}} g\right) d\left(x^{i} \otimes W_{i}\right),
$$

and we obtain the following properties:

Proposition 3.1. On the space $H_{1, W}\left(\mathbb{T}^{d}\right)$ we have

- (Poincaré's Inequality) Let $f \in H_{1, W}\left(\mathbb{T}^{d}\right)$. Then, there exists a finite constant $C$ such that

$$
\left\|f-\int_{\mathbb{T}^{d}} f d x\right\|_{L^{2}\left(\mathbb{T}^{d}\right)}^{2} \leq C \sum_{i=1}^{n} \int_{\mathbb{T}^{d}}\left(\partial_{W_{i}} f\right)^{2} d\left(x^{i} \otimes W_{i}\right):=C\left\|\nabla_{W} f\right\|_{L_{W}^{2}\left(\mathbb{T}^{d}\right)}^{2} .
$$

- (Rellich-Kondrachov's embedding) The embedding $H_{1, W}\left(\mathbb{T}^{d}\right) \subset \mathbb{L}^{2}\left(\mathbb{T}^{d}\right)$ is compact.

- Denote by $H_{W}^{-1}\left(\mathbb{T}^{d}\right)$ the dual space of $H_{1, W}\left(\mathbb{T}^{d}\right)$. Thus $f \in H_{W}^{-1}\left(\mathbb{T}^{d}\right)$ if and only if there exist functions $f_{0} \in L^{2}\left(\mathbb{T}^{d}\right)$, and $f_{k} \in L_{x^{k} \otimes W_{k}, 0}^{2}\left(\mathbb{T}^{d}\right)$, such that

$$
f=f_{0}-\sum_{i=1}^{d} \partial_{x_{i}} f_{i}
$$

in the sense that for $v \in H_{1, W}\left(\mathbb{T}^{d}\right)$

$$
(f, v)=\int_{\mathbb{T}^{d}} f_{0} v d x+\sum_{i=1}^{d} \int_{\mathbb{T}^{d}} f_{i}\left(\partial_{W_{i}} v\right) d\left(x^{i} \otimes W_{i}\right),
$$

with $(\cdot, \cdot)$ being the dual pairing. Furthermore,

$$
\|f\|_{H_{W}^{-1}}=\inf \left\{\left(\int_{\mathbb{T}^{d}} \sum_{i=0}^{d}\left|f_{i}\right|^{2} d x\right)^{1 / 2} ; \quad f \text { satisfies (3.3) }\right\} .
$$




\subsection{The elliptic equations}

In this subsection we investigate the solvability of uniformly elliptic generalized partial differential equations. Energy methods within Sobolev spaces are, essentially, the techniques exploited.

Let $A=\left(a_{i i}(x)\right)_{d \times d}, x \in \mathbb{T}^{d}$, be a mensurable diagonal matrix function satisfying the ellipticity condition, that is, there exists a constant $\theta>0$ satisfying

$$
\theta^{-1} \leq a_{i i}(x) \leq \theta
$$

for every $x \in \mathbb{T}^{d}$ and $i=1, \ldots, d$. To keep notation simple, we write $a_{i}(x)$ to mean $a_{i i}(x)$.

Our interest lies on solving the problem

$$
T_{\lambda} u=f
$$

on $u$, where $u: \mathbb{T}^{d} \rightarrow \mathbb{R}$, and $f: \mathbb{T}^{d} \rightarrow \mathbb{R}$ is given. Here $T_{\lambda}$ denotes the generalized elliptic operator

$$
T_{\lambda} u:=\lambda u-\nabla A \nabla_{W} u:=\lambda u-\sum_{i=1}^{d} \partial_{x_{i}}\left(a_{i}(x) \partial_{W_{i}} u\right) .
$$

The bilinear form $B_{\lambda}[\cdot, \cdot]$ associated with the elliptic operator $T_{\lambda}$ is given by

$$
B_{\lambda}[u, v]=\langle u, v\rangle_{1, W}=\lambda\langle u, v\rangle+\sum_{i=1}^{d} \int a_{i}(x)\left(\partial_{W_{i}} u\right)\left(\partial_{W_{i}} v\right) d\left(W_{i} \otimes x_{i}\right),
$$

where $u, v \in H_{1, W}\left(\mathbb{T}^{d}\right)$.

Let $f \in H_{W}^{-1}\left(\mathbb{T}^{d}\right)$. A function $u \in H_{1, W}\left(\mathbb{T}^{d}\right)$ is said to be a weak solution of the equation $T_{\lambda} u=f$ if

$$
B_{\lambda}[u, v]=(f, v) \text { for all } v \in H_{1, W}\left(\mathbb{T}^{d}\right)
$$

Denote by $H_{1, W}^{\perp}\left(\mathbb{T}^{d}\right)$ be the set of functions in $H_{1, W}\left(\mathbb{T}^{d}\right)$ which are orthogonal to the constant functions:

$$
H_{1, W}^{\perp}\left(\mathbb{T}^{d}\right)=\left\{f \in H_{1, W}\left(\mathbb{T}^{d}\right) ; \int_{\mathbb{T}^{d}} f d x=0\right\} .
$$

Proposition 3.2 (Energy estimates for $\lambda=0$ ). Let $B_{0}$ be the bilinear form on $H_{1, W}\left(\mathbb{T}^{d}\right)$ defined in (3.7) with $\lambda=0$. There exist constants $\alpha>0$ and $\beta>0$ such that for all $u, v \in H_{1, W}\left(\mathbb{T}^{d}\right)$,

$$
\left|B_{0}[u, v]\right| \leq \alpha\|u\|_{1, W}\|v\|_{1, W}
$$

and for all $u \in H_{1, W}^{\perp}$

$$
B_{0}[u, u] \geq \beta\|u\|_{1, W}^{2}
$$

The proof of this result follows from Poincaré's inequality and (3.4). 
Corollary 3.3. Let $f \in L^{2}\left(\mathbb{T}^{d}\right)$. There exists a weak solution $u \in H_{1, W}\left(\mathbb{T}^{d}\right)$ for the equation

$$
\nabla A \nabla_{W} u=f
$$

if and only if

$$
\int_{\mathbb{T}^{d}} f d x=0
$$

In this case, we have uniquenesses of the weak solutions if we disregard addition by constant functions. Also, let $u$ be the unique weak solution of (3.8) in $H_{1, W}^{\perp}\left(\mathbb{T}^{d}\right)$, then

$$
\|u\|_{1, W} \leq C\|f\|_{L^{2}\left(\mathbb{T}^{d}\right)},
$$

for some constant $C$ independent of $f$.

To prove this result we begin by noting that, from Proposition 3.2, $B$ satisfies the hypotheses of the Lax-Milgram's Theorem, [1, chapter 6]. This implies that there exists a weak solution $u \in H_{1, W}\left(\mathbb{T}^{d}\right)$ of (3.8). Since the function $v \equiv 1 \in H_{1, W}\left(\mathbb{T}^{d}\right)$, and $\partial_{W_{i}} 1=0$, we have from the definition of weak solution that

$$
\int_{\mathbb{T}^{d}} f d x=B_{0}[u, v]=0
$$

Furthermore, the Proposition 3.2 also implies that there is a $\beta>0$ such that

$$
\beta\|u\|_{1, W}^{2} \leq B_{0}[u, u]=\langle f, u\rangle \leq\|f\|_{L^{2}\left(\mathbb{T}^{d}\right)}\|u\|_{L^{2}\left(\mathbb{T}^{d}\right)} \leq\|f\|_{L^{2}\left(\mathbb{T}^{d}\right)}\|u\|_{1, W} .
$$

The existence of weak solutions and the bound $C$ in the statement of the Corollary follows from the previous expression.

Proposition 3.4 (Energy estimates for $\lambda>0$ ). Let $f \in L^{2}\left(\mathbb{T}^{d}\right)$ and $\lambda>0$. There exists $a$ unique weak solution $u \in H_{1, W}\left(\mathbb{T}^{d}\right)$ for the equation

$$
\lambda u-\nabla A \nabla_{W} u=f .
$$

This solution enjoys the following bounds

$$
\|u\|_{1, W} \leq C\|f\|_{L^{2}\left(\mathbb{T}^{d}\right)}
$$

for some constant $C>0$ independent of $f$, and

$$
\|u\| \leq \lambda^{-1}\|f\|_{L^{2}\left(\mathbb{T}^{d}\right)} .
$$

To obtain this result, note that an elementary computation shows that

$$
\left|B_{\lambda}[u, v]\right| \leq \alpha\|u\|_{1, W}\|v\|_{1, W} \quad \text { and } \quad B_{\lambda}[u, u] \geq \beta\|u\|_{1, W}^{2},
$$

where $\beta=\min \left\{\lambda, \theta^{-1}\right\}>0, \alpha=\max \{\lambda, \theta\}<\infty$ and $\theta$ is given in (3.4). The proof follows from Lax-Milgram's Theorem and an estimate similar to (3.9). 
Observe that, for $\lambda>0$, the operator $\lambda \mathbb{I}-\mathcal{L}_{W}^{A}: \mathcal{D}_{W} \rightarrow L^{2}\left(\mathbb{T}^{d}\right)$ is bijective. Therefore, the equation

$$
\lambda u-\nabla A \nabla_{W} u=f
$$

has strong solution in $\mathbb{D}_{W}$ if and only if $f \in\left(\lambda \mathbb{I}-\mathcal{L}_{W}^{A}\right)\left(\mathbb{D}_{W}\right)$, where $\mathbb{I}$ is the identity operator and $\left(\lambda \mathbb{I}-\mathcal{L}_{W}^{A}\right)\left(\mathbb{D}_{W}\right)$ stands for the range of $\mathbb{D}_{W}$ under the operator $\lambda \mathbb{I}-\mathcal{L}_{W}^{A}$. Moreover, this strong solution coincides with the weak solution obtained in Proposition 3.4.

\section{3 $W$-evolution equations}

We study a class of $W$-generalized PDEs that evolves in time: the parabolic equations. We begin by introducing the class of $W$-generalized parabolic equations we are interested. Then, we define what is meant by weak solution of such equations using the $W$-Sobolev spaces.

Fix $T>0$ and let $\left(B,\|\cdot\|_{B}\right)$ be a Banach space. We denote by $L^{2}([0, T], B)$ the Banach space of measurable functions $U:[0, T] \rightarrow B$ such that

$$
\|U\|_{L^{2}([0, T], B)}^{2}:=\int_{0}^{T}\left\|U_{t}\right\|_{B}^{2} d t<\infty .
$$

Let $A=A(t, x)$ be a diagonal matrix satisfying the ellipticity condition (3.4) for all $t \in[0, T]$, and let $\Phi:[l, r] \rightarrow \mathbb{R}$ be a continuously differentiable function such that

$$
B^{-1}<\Phi^{\prime}(x)<B
$$

for all $x$, where $B>0, l, r \in \mathbb{R}$ are constants. We will consider the equation

$$
\left\{\begin{array}{cl}
\partial_{t} u=\nabla A \nabla_{W} \Phi(u) & \text { in }(0, T] \times \mathbb{T}^{d}, \\
u=\gamma & \text { in }\{0\} \times \mathbb{T}^{d} .
\end{array}\right.
$$

where $u:[0, T] \times T^{d} \rightarrow \mathbb{R}$ is the unknown function, and $\gamma: \mathbb{T}^{d} \rightarrow \mathbb{R}$ is given.

We say that a function $\rho=\rho(t, x)$ is a weak solution of the problem (3.11) if:

- For every $H \in \mathbb{D}_{W}$ the following integral identity holds:

$$
\int_{\mathbb{T}^{d}} \rho(t, x) H(x) d x-\int_{\mathbb{T}^{d}} \gamma(x) H(x) d x=\int_{0}^{t} \int_{\mathbb{T}^{d}} \Phi(\rho(s, x)) \nabla A \nabla_{W} H(x) d x d s
$$

- $\Phi(\rho(\cdot, \cdot))$ and $\rho(\cdot, \cdot)$ belong to $L^{2}\left([0, T], H_{1, W}\left(\mathbb{T}^{d}\right)\right)$ :

$$
\int_{0}^{T}\|\Phi(\rho(s, x))\|_{L^{2}\left(\mathbb{T}^{d}\right)}^{2}+\left\|\nabla_{W} \Phi(\rho(s, x))\right\|_{L_{W}^{2}\left(\mathbb{T}^{d}\right)}^{2} d s<\infty
$$

and

$$
\int_{0}^{T}\|\rho(s, x)\|_{L^{2}\left(\mathbb{T}^{d}\right)}^{2}+\left\|\nabla_{W} \rho(s, x)\right\|_{L_{W}^{2}\left(\mathbb{T}^{d}\right)}^{2} d s<\infty
$$


We define the energy in $j$ th direction of a function $u$ as

$$
\begin{aligned}
\mathcal{Q}_{j}(u)= & \sup _{H \in \mathbb{D}_{W}}\left\{2 \int_{0}^{T} \int_{\mathbb{T}^{d}}\left(\partial_{x_{j}} \partial_{W_{j}} H\right)(s, x) u(s, x) d x d s\right. \\
& \left.-\int_{0}^{T} d s \int_{\mathbb{T}^{d}}\left[\partial_{W_{j}} H(s, x)\right]^{2} d\left(x^{j} \otimes W_{j}\right)\right\},
\end{aligned}
$$

and the total energy of a function $u$ as

$$
\mathcal{Q}(u)=\sum_{j=1}^{d} \mathcal{Q}_{j}(u) .
$$

There is a connection between the functions of finite energy and functions in the Sobolev space $H_{1, W}\left(\mathbb{T}^{d}\right)$. In fact, from [11, Lemma 4.1], a function $u \in L^{2}\left([0, T], L^{2}\left(\mathbb{T}^{d}\right)\right)$ has finite energy if and only if $u$ belongs to $L^{2}\left([0, T], H_{1, W}\left(\mathbb{T}^{d}\right)\right)$. In the case the energy is finite, we have

$$
\mathcal{Q}(u)=\int_{0}^{T}\left\|\nabla_{W} u\right\|_{L_{W}^{2}\left(\mathbb{T}^{d}\right)}^{2} d t .
$$

Moreover, we have uniqueness of weak solutions of the problem (3.11):

Lemma 3.5. Fix $\lambda>0$, two density profiles $\gamma^{1}, \gamma^{2}: \mathbb{T} \rightarrow[l, r]$ and denote by $\rho^{1}, \rho^{2}$ weak solutions of (3.11) with initial value $\gamma^{1}, \gamma^{2}$, respectively. Then,

$$
\left\langle\rho_{t}^{1}-\rho_{t}^{2}, \rho_{t}^{1, \lambda}-\rho_{t}^{2, \lambda}\right\rangle \leq\left\langle\gamma^{1}-\gamma^{2}, \gamma^{1, \lambda}-\gamma^{2, \lambda}\right\rangle e^{B \lambda t / 2}
$$

for all $t>0$. In particular, there exists at most one weak solution of (3.11).

The proof can be found in [11].

\section{HOMOGENIZATION OF RANDOM OPERATORS}

In this section we describe stochastic homogenization results for the $W$-generalized elliptic differential operator. This approach is closely related to the one considered in [10]. The focus of this approach is to study the asymptotic behavior of effective coefficients for a family of random difference schemes whose coefficients can be obtained by the discretization of random highcontrast lattice structures. The study of homogenization is motivated by several applications in mechanics, physics, chemistry and engineering. The details on this section can be found in [12].

\subsection{Discrete approximation}

Let $\mathbb{T}_{N}$ be the one-dimensional discrete torus with $N$ points:

$$
\mathbb{T}_{N}=\mathbb{R} / N \mathbb{Z} \simeq\{0,1, \ldots, N-1\} .
$$


Let, also, $\mathbb{T}_{N}^{d}=\mathbb{T}_{N} \times \ldots \times \mathbb{T}_{N}$ the $d$-dimensional discrete torus with $N^{d}$ points. Let $f$ : $\frac{1}{N} \mathbb{T}_{N}^{d} \rightarrow \mathbb{R}$ be a function and define the difference operators:

$$
\begin{aligned}
\partial_{x_{j}}^{N} f(x / N) & =N\left[f\left(\left(x+e_{j}\right) / N\right)-f(x / N)\right] \text { and } \\
\partial_{W_{j}}^{N} f(x / N) & =\frac{f\left(\left(x+e_{j}\right) / N\right)-f(x / N)}{W_{j}\left(\left(x+e_{j}\right) / N\right)-W_{j}(x / N)} .
\end{aligned}
$$

Consider $\nabla_{W}^{N} f=\left(\partial_{W_{1}}^{N} f, \ldots, \partial_{W_{d}}^{N} f\right)$ and $\nabla^{N} f=\left(\partial_{x_{1}}^{N} f, \ldots, \partial_{x_{d}}^{N} f\right)$.

We introduce now three inner products:

$$
\begin{gathered}
\langle f, g\rangle_{N}:=\frac{1}{N^{d}} \sum_{x \in \mathbb{T}_{N}^{d}} f(x) g(x), \\
\langle f, g\rangle_{W_{j}, N}:=\frac{1}{N^{d-1}} \sum_{x \in \mathbb{T}_{N}^{d}} f(x) g(x)\left(W\left(\left(x+e_{j}\right) / N\right)-W(x / N)\right), \\
\langle f, g\rangle_{1, W, N}:=\langle f, g\rangle_{N}+\sum_{j=1}^{d}\left\langle\partial_{W_{j}}^{N} f, \partial_{W_{j}}^{N} g\right\rangle_{W_{j}, N},
\end{gathered}
$$

and its induced norms:

$$
\|f\|_{L^{2}\left(\mathbb{T}_{N}^{d}\right)}^{2}=\langle f, f\rangle_{N}, \quad\|f\|_{L_{W_{j}}^{2}\left(\mathbb{T}_{N}^{d}\right)}^{2}=\langle f, f\rangle_{W_{j}, N} \text { and }\|f\|_{H_{1, W}\left(\mathbb{T}_{N}^{d}\right)}^{2}=\langle f, f\rangle_{1, W, N} .
$$

These norms are natural discretizations of the norms introduced in the previous sections.

Let $A=\left(a_{i j}\right)_{n \times n}$ be a diagonal matrix and let $T_{\lambda}^{N}$ denote the discrete generalized elliptic operator

$$
T_{\lambda}^{N} u:=\lambda u-\nabla^{N} A \nabla_{W}^{N} u,
$$

with

$$
\nabla^{N} A \nabla_{W}^{N} u:=\sum_{i=1}^{d} \partial_{x_{i}}^{N}\left(a_{i}(x / N) \partial_{W_{i}}^{N} u\right) .
$$

The bilinear form $B^{N}[\cdot, \cdot]$ associated with the elliptic operator $T_{\lambda}^{N}$ is given by

$$
\begin{gathered}
B^{N}[u, v]=\lambda\langle u, v\rangle_{N} \\
+\frac{1}{N^{d-1}} \sum_{i=1}^{d} \sum_{x \in \mathbb{T}_{N}^{d}} a_{i}(x / N)\left(\partial_{W_{i}}^{N} u\right)\left(\partial_{W_{i}}^{N} v\right)\left[W_{i}\left(\left(x_{i}+1\right) / N\right)-W_{i}\left(x_{i} / N\right)\right],
\end{gathered}
$$

where $u, v: N^{-1} \mathbb{T}_{N}^{d} \rightarrow \mathbb{R}$.

A function $u: N^{-1} \mathbb{T}_{N}^{d} \rightarrow \mathbb{R}$ is said to be a weak solution of the equation

$$
T_{\lambda}^{N} u=f
$$


where $u: N^{-1} \mathbb{T}_{N}^{d} \rightarrow \mathbb{R}$ is the unknown function, and $f: N^{-1} \mathbb{T}_{N}^{d} \rightarrow \mathbb{R}$ is given, if

$$
B^{N}[u, v]=\langle f, v\rangle_{N} \text { for all } v: N^{-1} \mathbb{T}_{N}^{d} \rightarrow \mathbb{R}
$$

We say that a function $f: N^{-1} \mathbb{T}_{N}^{d} \rightarrow \mathbb{R}$ belongs to the discrete space of functions orthogonal to the constant functions $H_{N}^{\perp}\left(\mathbb{T}_{N}^{d}\right)$ if

$$
\frac{1}{N^{d}} \sum_{x \in \mathbb{T}_{N}^{d}} f(x / N)=0 .
$$

Note that in the set of functions in $\mathbb{T}_{N}^{d}$ we have a "Dirac measure" concentrated in a point $x$ as a function: the function that takes value $N^{d}$ in $x$ and zero elsewhere. Therefore, we may integrate these weak solutions with respect to this function to obtain that every weak solution is, in fact, a strong solution. Moreover, many properties of the Lebesgue's measure also holds for the normalized counting measure. In particular, many results stated in Section 3 can be formuled and proved mutatis mutandis to this discrete setup.

Lemma 4.1. The equation

$$
\nabla^{N} A \nabla_{W}^{N} u=f
$$

has a weak solution $u: N^{-1} \mathbb{T}_{N}^{d} \rightarrow \mathbb{R}$ if and only if

$$
\frac{1}{N^{d}} \sum_{x \in \mathbb{T}_{N}^{d}} f(x)=0 .
$$

In this case we have uniqueness of the solution disregarding addition by constants. Moreover, if $u \in H_{N}^{\perp}\left(\mathbb{T}_{N}^{d}\right)$ we have the bound

$$
\|u\|_{H_{1, W}\left(\mathbb{T}_{N}^{d}\right)} \leq C\|f\|_{L^{2}\left(\mathbb{T}_{N}^{d}\right)} \text {, and }\|u\|_{L^{2}\left(\mathbb{T}_{N}^{d}\right)} \leq \lambda^{-1}\|f\|_{L^{2}\left(\mathbb{T}_{N}^{d}\right)},
$$

where $C>0$ does not depend on $f$ nor $N$.

Lemma 4.2. Let $\lambda>0$. There exists a unique weak solution $u: N^{-1} \mathbb{T}_{N}^{d} \rightarrow \mathbb{R}$ of the equation

$$
\lambda u-\nabla^{N} A \nabla_{W}^{N} u=f .
$$

Moreover,

$$
\|u\|_{H_{1, W}\left(\mathbb{T}_{N}^{d}\right)} \leq C\|f\|_{L^{2}\left(\mathbb{T}_{N}^{d}\right)} \text {, and }\|u\|_{L^{2}\left(\mathbb{T}_{N}^{d}\right)} \leq \lambda^{-1}\|f\|_{L^{2}\left(\mathbb{T}_{N}^{d}\right)},
$$

where $C>0$ does not depend neither on $f$ nor $N$.

Note that if $f \in L^{2}\left(\mathbb{T}^{d}\right)$ in Lemma 4.2, and $u$ is a weak solution of the problem (4.3), then following bound holds:

$$
\|u\|_{H_{1, W}\left(\mathbb{T}_{N}^{d}\right)} \leq C\|f\|_{L^{2}\left(\mathbb{T}^{d}\right)}
$$

since $\|f\|_{L^{2}\left(\mathbb{T}_{N}^{d}\right)} \rightarrow\|f\|_{L^{2}\left(\mathbb{T}^{d}\right)}$ as $N \rightarrow \infty$. 


\subsection{Connections between the discrete and continuous Sobolev spaces}

Given a function $f \in H_{1, W}\left(\mathbb{T}^{d}\right)$, we can define its restriction, $f_{N}$, to the lattice $N^{-1} \mathbb{T}_{N}^{d}$ as

$$
f_{N}(x)=f(x) \text { if } x \in N^{-1} \mathbb{T}_{N}^{d} .
$$

However, given a function $f: N^{-1} \mathbb{T}_{N}^{d} \rightarrow \mathbb{R}$ it is not straightforward how to define an extension belonging to $H_{1, W}\left(\mathbb{T}^{d}\right)$. To do so, we need the definition of $W$-interpolation, which we give below.

Let $f_{N}: N^{-1} \mathbb{T}_{N} \rightarrow \mathbb{R}$ and $W: \mathbb{R} \rightarrow \mathbb{R}$, a strictly increasing right continuous function with left limits (càdlàg), and periodic. The $W$-interpolation $f_{N}^{*}$ of $f_{N}$ is given by:

$$
\begin{aligned}
f_{N}^{*}(x+t):= & \frac{W((x+1) / N)-W((x+t) / N)}{W((x+1) / N)-W(x / N)} f(x) \\
& +\frac{W((x+t) / N)-W(x / N)}{W((x+1) / N)-W(x / N)} f(x+1),
\end{aligned}
$$

for $0 \leq t<1$. Note that

$$
\frac{\partial f_{N}^{*}}{\partial W}(x+t)=\frac{f(x+1)-f(x)}{W((x+1) / N)-W(x / N)}=\partial_{W}^{N} f(x) .
$$

Using the standard construction of $d$-dimensional linear interpolation, it is possible to define the $W$-interpolation of a function $f_{N}: \mathbb{T}_{N}^{d} \rightarrow \mathbb{R}$, with $W(x)=\sum_{i=1}^{d} W_{i}\left(x_{i}\right)$ as defined in (2.1).

We now establish the connection between the discrete and continuous Sobolev spaces by showing how a sequence of functions defined in $\mathbb{T}_{N}^{d}$ can converge to a function in $H_{1, W}\left(\mathbb{T}^{d}\right)$.

We say that a family $f_{N} \in L^{2}\left(\mathbb{T}_{N}^{d}\right)$ converges strongly (resp. weakly) to the function $f \in L^{2}\left(\mathbb{T}^{d}\right)$ as $N \rightarrow \infty$, if $f_{N}^{*}$ converges strongly (resp. weakly) to the function $f$. From now on we will omit the symbol "*" in the $W$-interpolated function, and denote them simply by $f_{N}$.

The convergence in $H_{W}^{-1}\left(\mathbb{T}^{d}\right)$ can be defined in terms of duality. Namely, we say that a functional $f_{N}$ on $\mathbb{T}_{N}^{d}$ converges to $f \in H_{W}^{-1}\left(\mathbb{T}^{d}\right.$ ) weakly (resp. strongly) if for any sequence of functions $u_{N}: \mathbb{T}_{N}^{d} \rightarrow \mathbb{R}$ and $u \in H_{1, W}\left(\mathbb{T}^{d}\right)$ such that $u_{N} \rightarrow u$ weakly (resp. strongly) in $H_{1, W}\left(\mathbb{T}^{d}\right)$, we have

$$
\left(f_{N}, u_{N}\right)_{N} \longrightarrow(f, u), \quad \text { as } N \rightarrow \infty
$$

\subsection{Random environment}

In this subsection we introduce the statistically homogeneous rapidly oscillating coefficients that will be used to define the random $W$-generalized difference elliptic operators.

Let $(\Omega, \mathcal{F}, \mu)$ be a standard probability space and $\left\{T_{x}: \Omega \rightarrow \Omega ; x \in \mathbb{Z}^{d}\right\}$ be a group of $\mathcal{F}$ measurable and ergodic transformations which preserve the measure $\mu$ :

- $T_{x}: \Omega \rightarrow \Omega$ is $\mathcal{F}$-measurable for all $x \in \mathbb{Z}^{d}$, 
- $\mu\left(T_{x} \mathbf{A}\right)=\mu(\mathbf{A})$, for any $\mathbf{A} \in \mathcal{F}$ and $x \in \mathbb{Z}^{d}$,

- $T_{0}=I, \quad T_{x} \circ T_{y}=T_{x+y}$,

- For any $f \in L^{1}(\Omega)$ such that $f\left(T_{x} \omega\right)=f(\omega) \quad \mu$-a.s for each $x \in \mathbb{Z}^{d}$, is equal to a constant $\mu$-a.s.

Note that the last condition implies that the group $T_{x}$ is ergodic. Let us now introduce the vectorvalued $\mathcal{F}$-measurable functions $\left\{a_{j}(\omega) ; j=1, \ldots, d\right\}$ such that there exists $\theta>0$ with

$$
\theta^{-1} \leq a_{j}(w) \leq \theta
$$

for all $\omega \in \Omega$ and $j=1, \ldots, d$. Then, define the diagonal matrices $A^{N}$ whose elements are given by

$$
a_{j j}^{N}(x):=a_{j}^{N}=a_{j}\left(T_{N x} \omega\right), x \in T_{N}^{d}, \quad j=1, \ldots, d .
$$

Let $\lambda>0$, and let $f_{N}$ be a functional on the space of functions $h_{N}: \mathbb{T}_{N}^{d} \rightarrow \mathbb{R}, f \in H_{W}^{-1}\left(\mathbb{T}^{d}\right)$. Let, also, $u_{N}$ be the unique weak solution of

$$
\lambda u_{N}-\nabla^{N} A^{N} \nabla_{W}^{N} u_{N}=f_{N},
$$

and $u_{0}$ be the unique weak solution of

$$
\lambda u_{0}-\nabla A \nabla_{W} u_{0}=f .
$$

We say that the diagonal matrix $A$ is a homogenization of the sequence of random matrices $A^{N}$ if the following conditions hold:

- For each sequence $f_{N} \rightarrow f$ in $H_{W}^{-1}\left(\mathbb{T}^{d}\right), u_{N}$ converges weakly in $H_{1, W}$ to $u_{0}$, when $N \rightarrow \infty$;

- $a_{i}^{N} \partial_{W_{i}}^{N} u^{N} \rightarrow a_{i} \partial_{W_{i}} u$, weakly in $L_{x^{i} \otimes W_{i}}^{2}\left(\mathbb{T}^{d}\right)$ when $N \rightarrow \infty$.

The following homogenization theorem is proved in [12]:

Theorem 4.3. Let $A^{N}$ be a sequence of ergodic random matrices, such as the one that defines our random environment. Then, almost surely, $A^{N}(\omega)$ admits a homogenization, where the homogenized matrix A does not depend on the realization $\omega$.

Note that if $u \in \mathbb{D}_{W}$ is a strong solution (or weak) of

$$
\lambda u-\nabla A \nabla_{W} u=f
$$

and $u_{N}$ is strong solution of the discrete problem

$$
\lambda u_{N}-\nabla^{N} A^{N} \nabla_{W}^{N} u_{N}=f
$$

then, the homogenization theorem also holds, that is, $u_{N}$ also converges weakly in $H_{1, W}$ to $u$. 
RESUMO. Neste artigo discutimos recentes resultados sobre uma generalização do Laplaciano. Mais precisamente, fixe uma função $W\left(x_{1}, \ldots, x_{d}\right)=\sum_{k=1}^{d} W_{k}\left(x_{k}\right)$, onde cada $W_{k}: \mathbb{R} \rightarrow \mathbb{R}$ é uma função contínua á direita com limites a esquerda e estritamente crescente. Usando $W$, construímos o laplaciano generalizado $\mathcal{L}_{W}=\sum_{i=1}^{d} \partial_{x_{i}} \partial_{W_{i}}$, onde $\partial_{W_{i}}$ denota o operador diferencial induzido por $W_{i}$. Apresentamos resultados sobre propriedades espectrais de $\mathcal{L}_{W}$, espaços de Sobolev induzidos por $\mathcal{L}_{W}$ (espaços $W$-Sobolev), equações diferenciais parciais generalizadas, equações diferenciais estocásticas e homogeinização estocástica.

Palavras-chave: Espaços $W$-Sobolev, Laplaciano generalizado, Homogeinização, Equações diferenciais parciais.

\section{REFERENCES}

[1] L. Evans. Partial differential equation. AMS (1998).

[2] A. Faggionato, M. Jara \& C. Landim. Hydrodynamic behavior of one dimensional subdiffusive exclusion processes with random conductances. Probability Theory and Related Fields, 144 (2009), 633-667.

[3] J. Farfan, A.B. Simas \& F.J. Valentim. Equilibrium fluctuations for exclusion processes with conductances in random environments. Stochastic Processes and their Applications, 120 (2010), 1535-1562.

[4] W. Feller. On Second Order Differential Operators. Annals of Mathematics, 61(1) (1955), 90-105.

[5] W. Feller. Generalized second order differential operators and their lateral conditions. Illinois J. Math., 1(4) (1957), 459-504.

[6] T. Franco \& C. Landim. Hydrodynamic limit of gradient exclusion processes with conductances. Archive for Rational Mechanics and Analysis, (Print), 195(2009), 409-439.

[7] M. Jara, C. Landim \& A. Teixeira. Quenched scaling limits of trap models. Annals of Probability, 39(2011), 176-223.

[8] J.-U. Löbus. Generalized second order differential operators. Math. Nachr., 152 (1991), 229-245.

[9] P. Mandl. Analytical treatment of one-dimensional Markov processes. Grundlehren der mathematischen Wissenschaften, 151. Springer-Verlag, Berlin (1968).

[10] A. Piatnitski \& E. Remy. Homogenization of Elliptic Difference Operators. SIAM J. Math. Anal., 33 (2001), 53-83.

[11] A.B. Simas \& F.J. Valentim. W-Sobolev spaces. Journal of Mathematical Analysis and Applications, 382(1) (2011), 214-230.

[12] A.B. Simas \& F.J. Valentim. Homogenization of second-order generalized elliptic operators, submitted for publication.

[13] F.J. Valentim. Hydrodynamic limit of a $d$-dimensional exclusion process with conductances. Ann. Inst. H. Poincaré Probab. Statist., 48(1) (2012), 188-211.

[14] E. Zeidler. Applied Functional Analysis. Applications to Mathematical Physics. Applied Mathematical Sciences, 108. Springer-Verlag, New York (1995). 